\title{
An Adaptive Nonlinear Control for Gyro Stabilized Platform Based on Neural Networks and Disturbance Observer
}

\author{
Jiancheng Fang and Rui Yin \\ Fundamental Science on Novel Inertial Instrument \& Navigation System Technology Laboratory, \\ Beijing University of Aeronautics and Astronautics, Beijing 100191, China \\ Correspondence should be addressed to Rui Yin; yinruibuaa@163.com
}

Received 3 July 2014; Revised 30 October 2014; Accepted 10 November 2014; Published 25 November 2014

Academic Editor: Vu Ngoc Phat

Copyright (C) 2014 J. Fang and R. Yin. This is an open access article distributed under the Creative Commons Attribution License, which permits unrestricted use, distribution, and reproduction in any medium, provided the original work is properly cited.

\begin{abstract}
In order to improve the tracking performance of gyro stabilized platform with disturbances and uncertainties, an adaptive nonlinear control based on neural networks and reduced-order disturbance observer for disturbance compensation is developed. First the reduced-order disturbance observer estimates the disturbance directly. The error of the estimated disturbance caused by parameter variation and measurement noise is then approximated by neural networks. The phase compensation is also introduced to the proposed control law for the desired sinusoidal tracking. The stability of the proposed scheme is analyzed by the Lyapunov criterion. Experimental results show the validity of the proposed control approach.
\end{abstract}

\section{Introduction}

In order to obtain high resolution and definition imaging for many applications including surveillance, target tracking, and missile guidance, gyro stabilized platform (GSP) is used to stabilize and point the line-of-sight (LOS) of cameras, sensors, or other payload [1-6]. So, the tracking and stabilizing accuracy of GSP must be improved for high quality imaging. However, in a practical GSP system, the motion precision may seriously be degraded due to large quantities of disturbances and uncertainties, for example, unmodeled dynamics, parameter variation, friction force, imbalance, cable and spring torques, coupling torques, gyro and sensor noise, gear reactions, and load disturbances [1].

Therefore, to compensate for disturbances of GSP, different control strategies have been developed [7-11]. In [7], a reduced-order observer was designed according to linear control theory for an inertially stabilized line-of-sight control system. The method can be simply completed but requires an accurate system model to achieve satisfactory performance. The disturbance observer based internal-loop compensator was introduced in [8] to estimate the disturbance of GSP. However, this method is also dependent on the accurate system model. An extended-state-observer was used to estimate disturbance for a floated inertial platform in [9].
Unmodeled dynamics, parameters uncertainty, and sensor noise considered as the internal disturbance are estimated together with the external disturbance and are compensated to the control system as feed-forward control. However, due to the extension of the dimension of the system and the need of adjusting many parameters, the method is complicated and it is hard to obtain optimal parameters tuning. In [10], based on PI disturbance observer (DOB), an integral sliding mode controller (SMC) was presented for three-axis inertial platform. The method combines DOB with SMC to improve the precision of system, but the parameters of control system are without autotuning in the process of control. In order to attenuate the platform disturbance, two-degree-of-freedom internal model controller (IMC) was used in [11]. The IMC method has robustness on parameter perturbation, but the disturbance structure is needed to be known. Since neural networks $(\mathrm{NN})$ have distinct advantages of learning and approximating nonlinear functions and are independent of the system model or disturbance structure, they have received considerable attention in control systems for modeling and compensation purposes [12-16].

An adaptive nonlinear control using $\mathrm{NN}$ and reducedorder disturbance observer is proposed for disturbance compensation in this paper. All disturbances including friction force, imbalance, cable and spring torques, coupling torques, 


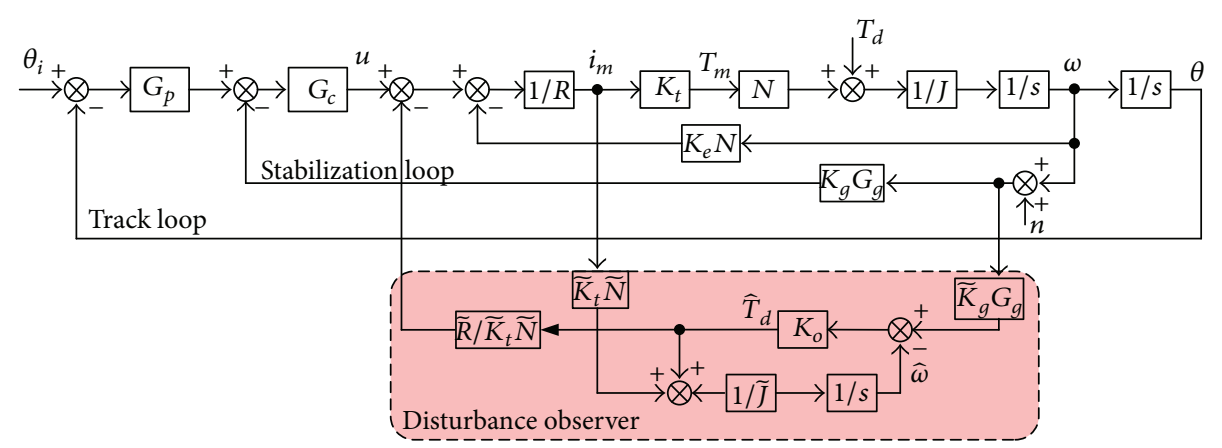

FIgURE 1: The single-axis GSP control system combined with a single-state observer block diagram.

load disturbances and equivalent disturbance caused by unmodeled dynamics, parameter variation, gyro and sensor noise, and gear reactions are considered a total disturbance, which is estimated by the reduced-order disturbance observer first. The error of the estimated disturbance is then approximated by NN. Phase compensation is introduced in the proposed control law for the desired sinusoidal tracking. The stability of the proposed scheme is analyzed by the Lyapunov criterion. Experimental results show that the tracking performance can be improved significantly with the use of the NN + DOB as compared to the case only with DOB compensation.

This paper is organized as follows. First, in Section 2, the system of GSP is modeled with the introduction of basic reduced-order $\mathrm{DOB}$, and noise coupling characteristics and sensitivity to parameters variation of the DOB are analyzed. Then, Section 3 is devoted to designing adaptive nonlinear controller for disturbance compensation. Experiments are developed in Section 4. Finally, Section 5 concludes this paper.

\section{Statement of the Problem}

In this section, some preliminaries are introduced first, including GSP model and basic reduced-order DOB design, and then noise coupling characteristics and sensitivity to parameters variation of the $\mathrm{DOB}$ are analyzed by the simulation results, to facilitate the proposal of the new methods.

2.1. GSP Model. The assumptions made for the development of GSP model are as follows: (1) motor inductance is ignored and (2) the gimbal is considered rigid. Then, the one gimbal model of GSP can be expressed as follows:

$$
\begin{gathered}
J \dot{\omega}=N T_{m}+T_{d}, \\
T_{m}=K_{t} i_{m}, \\
i_{m}=\frac{\left(u-K_{e} N \omega\right)}{R},
\end{gathered}
$$

where $J$ is the moment of inertia of gimbal; $u$ is the motor input voltage; $K_{t}$ is the motor torque constant; $K_{e}$ is the back-EMF coefficient; $R$ is the motor resistance; $i_{m}$ is the motor current; $T_{m}$ is the command torque from the gimbal drive motor; $N$ is the gear ratio; $\omega$ is the gimbal rate relative to the inertial coordinate; and $T_{d}$ is the torque-equivalent representation of all disturbance torques, which can disrupt the behavior of the gimbal system.

2.2. Basic Reduced-Order DOB Design. Figure 1 shows the block diagram of single-axis GSP control system combined with a single-state observer [7]. The control system is configured as a high bandwidth negative-feedback rate loop inside a lower bandwidth negative-feedback pointing or tracking position loop. The two loops, the track loop and the rate loop, must be stable, responsive to command inputs, and insensitive to disturbances, noise, and parameters variation. Here, $\widehat{T}_{d}$ is estimated disturbance torque. $K_{o}$ is observer gain. $K_{g}$ is gyro scale factor. $n$ is gyro measurement noise. $\omega_{i}$ is rate command. $\theta_{i}$ is angle command. $G_{c}$ is the feedback controller for rate loop. $G_{p}$ is the feedback controller for position loop. $G_{g}$ is the transfer function for the gyroscope. $\widehat{\omega}$ is the estimated angle velocity. $s$ is the Laplace variable. $\widetilde{J}$, $\widetilde{K}_{t}, \widetilde{N}, \widetilde{R}$, and $\widetilde{K}_{g}$ denote the best known values available for $J, K_{t}, N, R$, and $K_{g}$, respectively.

(1) Disturbance Rejection Characteristics. The disturbance rejection ratio $\theta /\left(T_{d} / J\right)$, which is used to estimate the angular motion $\theta$ due to the disturbance torque, can directly reflect the disturbance rejection characteristics of control system. The nature and quality of the disturbance rejection with different observer gains are interesting, so the simulation for the disturbance rejection characteristics is under the assumption that there is no parameters variation and $n=$ 0 . The parameters of single-axis GSP control system for simulation are listed in Table 1; the feedback controllers $G_{p}=$ $G_{c}=K_{p}=10$; the transfer function $G_{g}=1 / K_{g}$; angle command $\theta_{i}=\sin t$. The observer gain $K_{o}$ is selected as 0,100 , 500 , and 1000, respectively. The disturbance torque $T_{d}=$ $20 \sin \left(\omega_{f} t\right)$, with $\omega_{f}$ selected as $0.5,5$, and 50 , respectively. Figure 2 shows the frequency response of disturbance rejection transfer function with different observer gains, at the angle displacement, which is $\left(\theta_{i}-\theta\right) /\left(T_{d} / J\right)$. Figure 3 shows the angle displacement and the estimated disturbance torque $\widehat{T}_{d}$ with different observer gains under the condition of $T_{d}=$ $20 \sin (5 t)$. Figure 4 shows the angle displacement and the estimated disturbance torque error $\left(T_{d}-\widehat{T}_{d}\right)$ with different disturbance torque under the condition of $K_{o}=500$.

Disturbance rejection ability is enhanced with $K_{o}$ at lower frequencies. The higher the value of $K_{o}$, the less the error 
TABle 1: Parameters of single-axis GSP system.

\begin{tabular}{lcc}
\hline Moment of inertia & $\mathrm{N} \cdot \mathrm{m} \cdot \mathrm{s}^{2} / \mathrm{rad}$ & 3 \\
Back-EMF coefficient & $\mathrm{V} \cdot \mathrm{s} / \mathrm{rad}$ & 1.03 \\
Motor torque constant & $\mathrm{N} \cdot \mathrm{m} / \mathrm{A}$ & 1.03 \\
Motor resistance & $\Omega$ & 9 \\
Gear ratio & & 5.24 \\
Gyro scale factor & $\mathrm{V} / \mathrm{rad} / \mathrm{s}$ & 0.63 \\
\hline
\end{tabular}

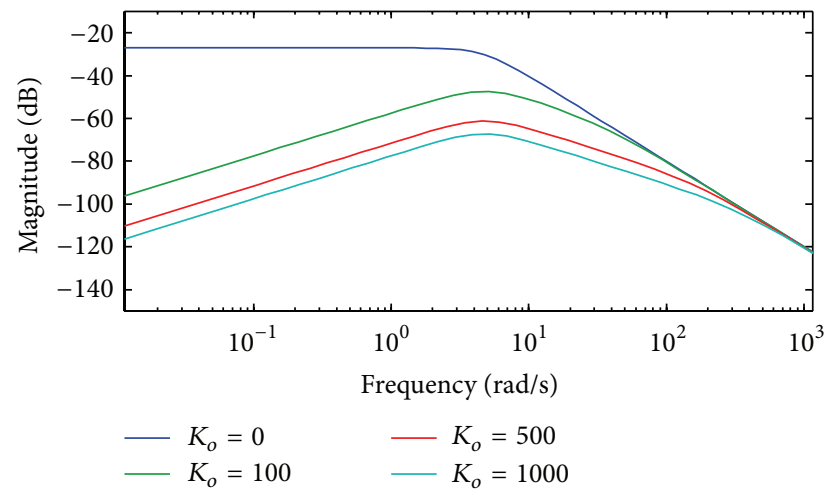

FIGURE 2: The frequency response of disturbance rejection transfer function with different observer gains, at the angle displacement.

of the estimated disturbance torque. However, at higher frequencies, the reduced-order observer has less effect on disturbance rejection characteristics. With the increment of disturbance frequency, the estimated disturbance torque error increases significantly. The disturbance rejection is mainly dependent on the basic control structure.

(2) Noise Coupling. Fiber optic gyroscope noise, which always exists in real GSP control system, is complicated and consisted of angular random walk, bias instability, rate random walk, rate ramp, quantization noise, exponential correlation noise, sinusoidal noise, and so forth. The expression of gyro noise is simplified in this paper as follows:

$$
\begin{gathered}
\omega_{o}(t)=\omega(t)+n(t), \\
n(t)=\varepsilon_{L}(t)+\varepsilon_{H}(t), \\
\varepsilon_{H}(t)=A_{H} \sin \left(2 \pi f_{H} t+\theta_{0}\right),
\end{gathered}
$$

where $\omega_{o}$ is the measured gyro rate; $\varepsilon_{L}$ is low frequency noise; $\varepsilon_{H}$ is high frequency noise; $A_{H}, f_{H}$, and $\theta_{0}$ are the amplitude, frequency, and initial phase of high frequency noise, respectively.

The following simulations are under the assumption that there is no parameters variation and $T_{d}=0$. Figure 5 shows the frequency response of noise coupling transfer function with different observer gains, at the angle displacement, which is $\left(\theta_{i}-\theta\right) / n$. Figure 6 shows the angle displacement with different observer gains under high frequency noise $n(t)=\sin (50 t)$ and low frequency noise $n(t)=1$, respectively.

At higher frequencies measurement noise, with the increment of $K_{o}$, the measurement noise couples more deeply, which leads to more angle displacement, while, at lower frequencies measurement noise, the observer has less effect on noise coupling, and a large angle error emerges because of the lower frequencies measurement noise.

(3) Variation of Parameters. The reduced-order observer shown in Figure 1 needs the most approximate parameter values of the single-axis GSP system, such as $\widetilde{J}, \widetilde{K} t, \widetilde{N}, \widetilde{R}$, and $\widetilde{K}_{g}$. In these parameters, $\widetilde{K}_{t}$ and $\widetilde{R}$ are the motor parameter, $\widetilde{K}_{g}$ is the gyro parameter, and $\widetilde{N}$ is the gear parameter, which have less parameter fluctuations than the gimbal inertia $\widetilde{J}$, for the whole system includes many components, and the gimbal inertia is difficult to confirm by measurement. So, the effect of the variation of the gimbal inertia on the disturbance rejection is mainly analyzed. The difference between the estimated and the actual inertia is $\Delta J$, which may be assumed to be known within about $\pm 20 \%$. Figure 7 shows the estimated disturbance torque with and without parameter variation under the condition of $K_{o}=100$. Figure 8 shows the error of estimated disturbance torque with different observer gains, under the condition of $\Delta J / J=20 \%$.

The parameter variations seriously reduce the accuracy of the estimated disturbance torque, which has no obvious change with different observer gains.

\section{Nonlinear Control for the GSP System}

In this section, in order to overcome the problem of low frequency gyro noise and parameter variation, a method of phase compensation is proposed first and, then, an adaptive nonlinear control for disturbance compensations is derived.

3.1. Phase Compensation. The block diagram of the singleaxis GSP control system without disturbance is shown in Figure 9.

The transfer function of the system can be derived as follows:

$$
\begin{aligned}
& G(s)=\frac{\theta(s)}{\theta_{i}(s)} \\
&=(1)\left(\frac{J R}{K_{t} N G_{p} G_{c}} s^{2}+\left(\frac{K_{e} N}{G_{p} G_{c}}+\frac{1}{G_{p}}\right) s+1\right)^{-1}, \\
& 20 \lg \mid G(j \omega) \mid \\
&= 20 \lg \\
& \times\left(( 1 ) \left(\left(1-\frac{J R}{K_{t} N G_{p} G_{c}} \omega^{2}\right)^{2}\right.\right. \\
&\left.\left.\left.+\left(\frac{K_{e} N}{G_{p} G_{c}}+\frac{1}{G_{p}}\right)^{2} \omega^{2}\right)^{1 / 2}\right)^{-1}\right), \\
& \angle G(j \omega)=\arctan \left(-\frac{\left(K_{t} K_{e} N^{2}+G_{c} K_{t} N\right) \omega}{G_{c} G_{p} K_{t} N-J R \omega^{2}}\right) .
\end{aligned}
$$




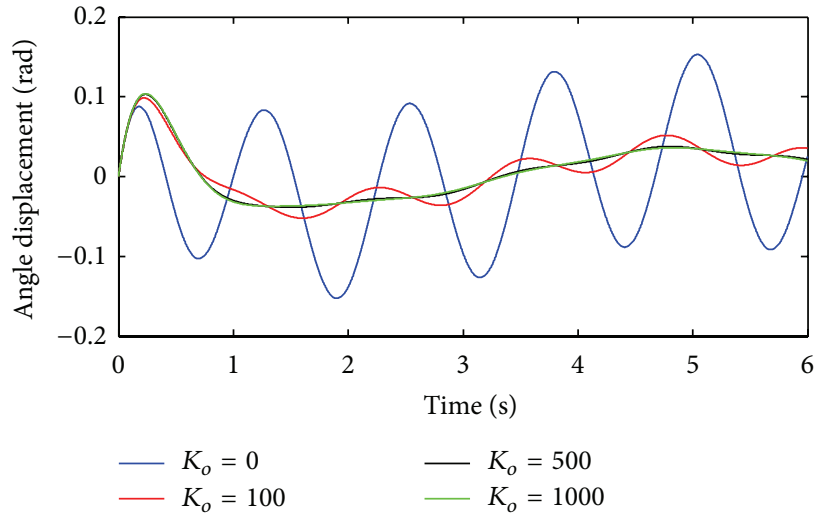

(a)

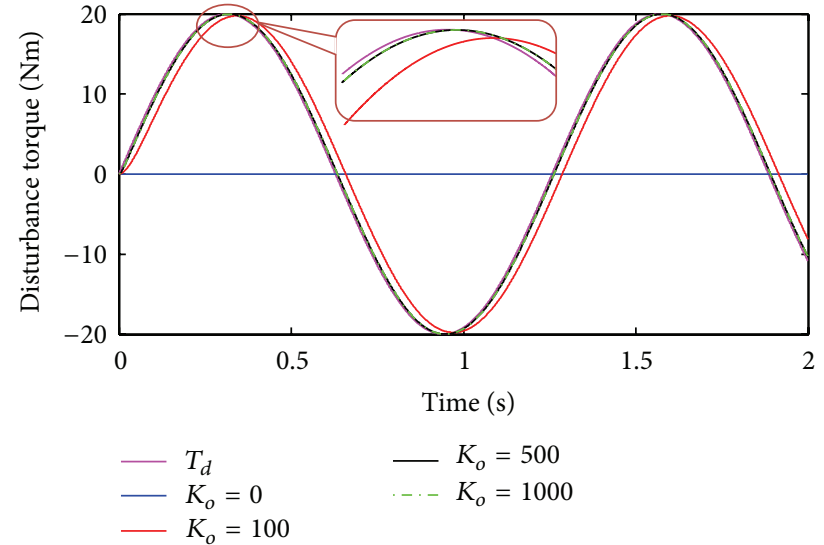

(b)

Figure 3: The angle displacement and the estimated disturbance torque $\widehat{T}_{d}$ with different observer gains under the condition of $T_{d}=$ $20 \sin (5 t)$.

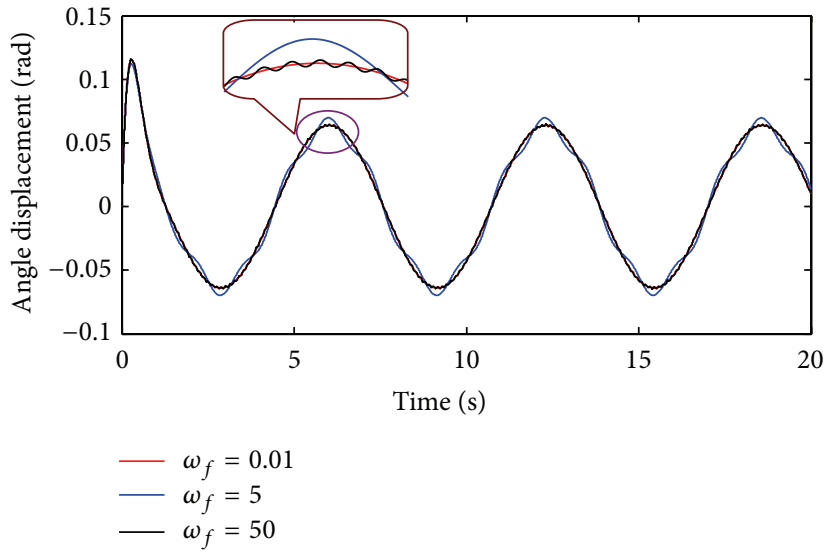

(a)

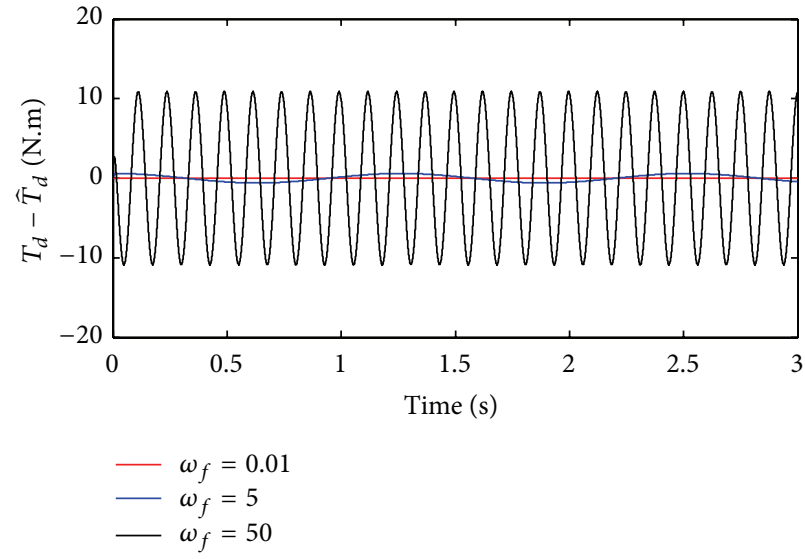

(b)

FIGURE 4: The angle displacement and the estimated disturbance torque error with different disturbance torque under the condition of $K_{o}=$ 500.

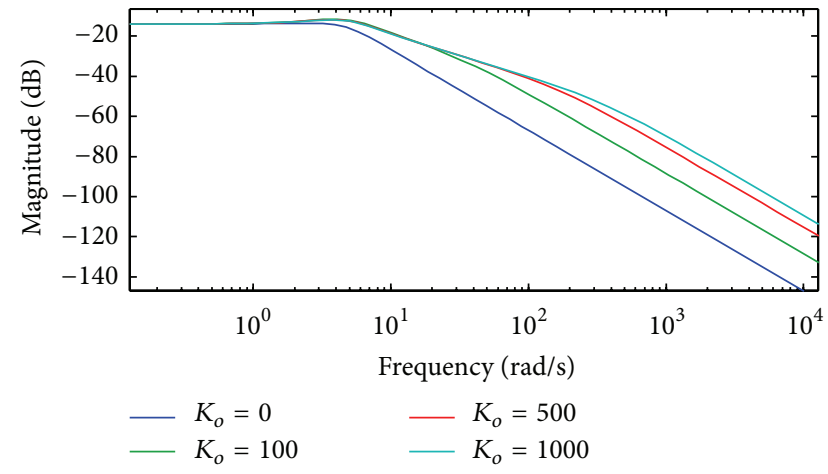

FIGURE 5: The frequency response of noise coupling transfer function with different observer gains, at the angle displacement.

Let $K_{t}=K_{e}=1.03, N=5.24, R=9, G_{p}=3, G_{c}=135.5$, and $J=3$. The frequency response figure of the transfer function $\left(\theta / \theta_{i}\right)$ is shown in Figure 10. As the frequency of angle command is increasing, the phase delay is obvious, so compensating the phase is necessary for tracking system.

Define the tracking error as $e_{1}=\theta_{i}-\theta$ and the velocity error as $e_{2}=\omega_{d}-\omega$, and $\omega_{d}=\dot{\theta}_{i}+G_{p} e_{1}$. The following control law for phase compensation is proposed, as shown in Figure 11:

$$
u=\frac{R}{K_{t} N}\left(J \ddot{\theta}_{i}+J G_{p}\left(\dot{\theta}_{i}-\omega\right)+\frac{K_{t} K_{e} N^{2}}{R} \omega+G_{c} e_{2}\right)
$$

The transfer function of the system with the control law (4) can be obtained as follows, and the system gets an ideal result theoretically:

$$
G(s)=\frac{\theta(s)}{\theta_{i}(s)}=1
$$




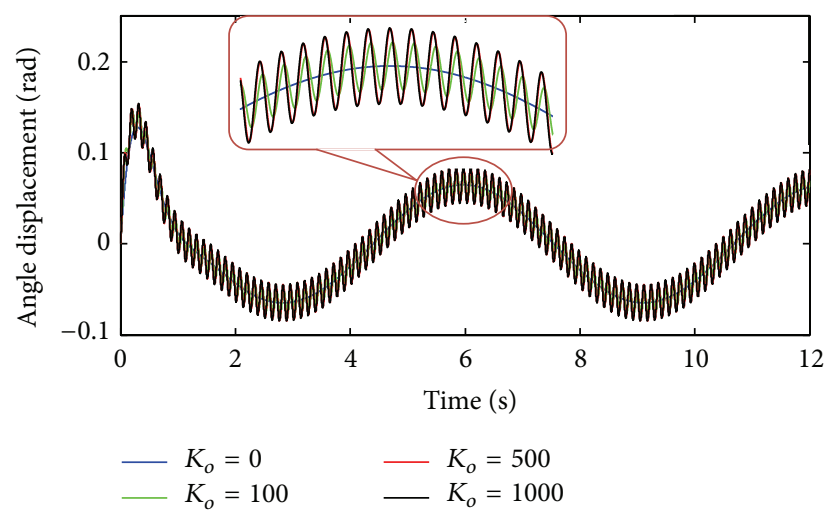

(a) $n(t)=\sin (50 t)$

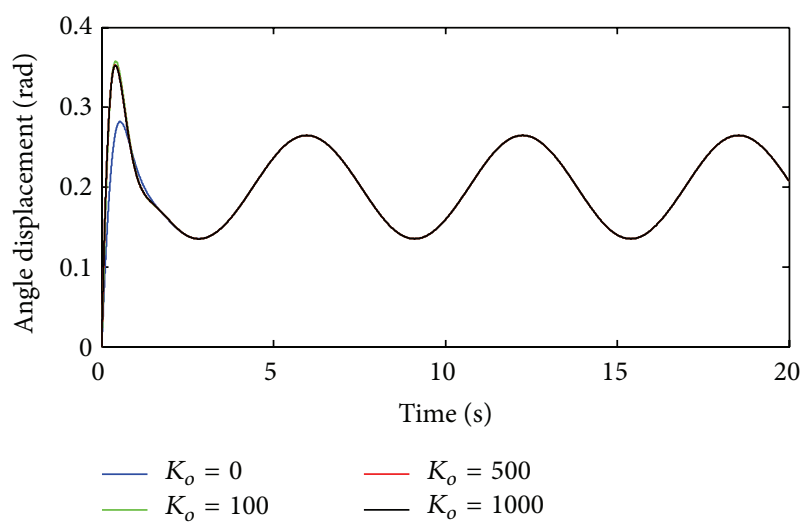

(b) $n(t)=1$

FIgURE 6: The angle displacement with different observer gains under high frequency and low frequency noise, respectively.

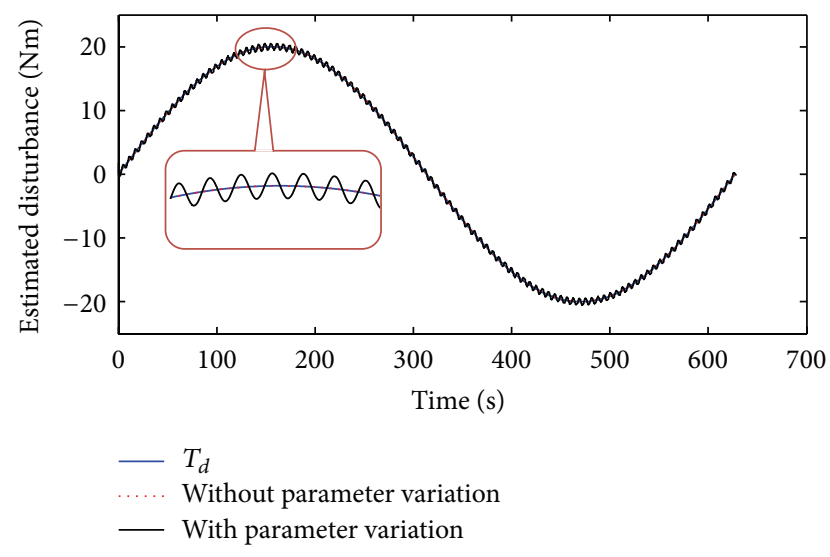

FIGURE 7: The estimated disturbance torque with and without parameter variation under the condition of $K_{o}=100$.

3.2. Disturbance Compensation. Differentiating the velocity error $e_{2}$ and using (1) and (4), the single-axis GSP control system dynamics can be expressed as

$$
J \dot{e}_{2}=J \ddot{\theta}_{i}+J G_{p} \dot{\theta}_{i}+\left(\frac{K_{e} K_{t} N^{2}}{R}-J G_{p}\right) \omega-\frac{K_{t} N}{R} u-T_{d}
$$

The effect of all disturbance torques $T_{d}$ is too complicated to be modeled, so the common compensation based on disturbance model is hardly complemented in this system. The reduced-order disturbance observer and neural networks compensator will be used to design a nonlinear controller for disturbance compensation. The disturbance is estimated by the reduced-order disturbance observer, and the neural networks compensator based on radial basis function (RBF) approximates the error of estimated disturbance caused by parameter variations, lower frequencies measurement noise, and so forth.

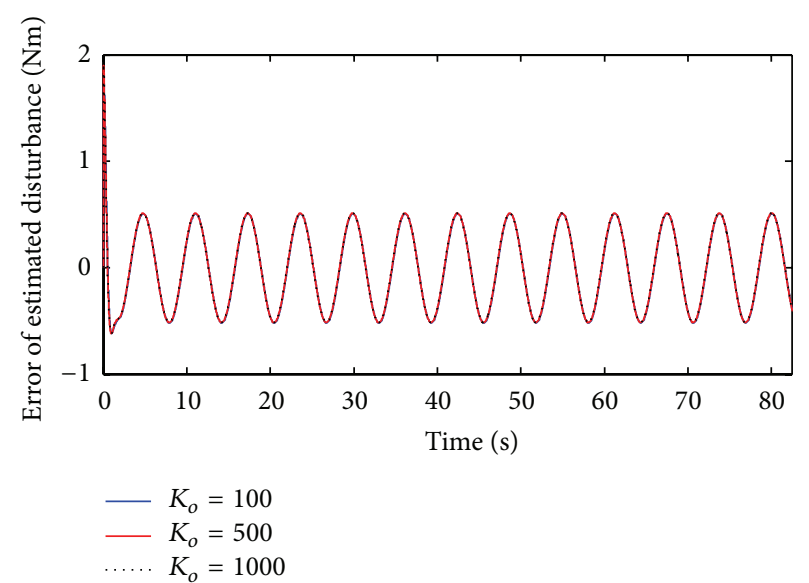

FIGURE 8: The error of estimated disturbance torque with different observer gains, under the condition of $\Delta J / J=20 \%$.

As shown in Figure 1, the estimated disturbance $\widehat{T}_{d}$ can be expressed as

$$
\begin{gathered}
\widehat{T}_{d}=K_{o}(\omega+n-\widehat{\omega}), \\
\widetilde{J} \dot{\hat{\omega}}=i_{m} \widetilde{K}_{t} \widetilde{N}+\widehat{T}_{d} .
\end{gathered}
$$

Define the disturbance estimated error as $e_{d}=T_{d}-\widehat{T}_{d}$. $\mathrm{NN}$ compensator based on RBF is used to approximate it as

$$
\widehat{e}_{d}^{*}=e_{d}=W^{* T} \phi(x)-\varepsilon,
$$

where $\widehat{e}_{d}^{*}$ denotes the optimal estimate value of $e_{d} ; W^{*} \in R^{m}$ is the optimal weight vector with the node number $m>1$; $\Phi(x) \in R^{m}$ is chosen as the commonly used Gaussian radial basis functions; $x=\omega$ is the network input vector; and $-\varepsilon$ is the NN approximation error satisfying $|\varepsilon| \leq \varepsilon_{M}$ with $\varepsilon_{M}>0$.

Since the ideal weights $W^{*}$ are unknown, let $\widehat{W}$ be the estimates of $W^{*}$; thus, the $\mathrm{NN}$ compensator is designed as

$$
\widehat{e}_{d}=\widehat{W}^{T} \phi(x),
$$

where $\widehat{e}_{d}$ is the estimated value of $e_{d}$. 


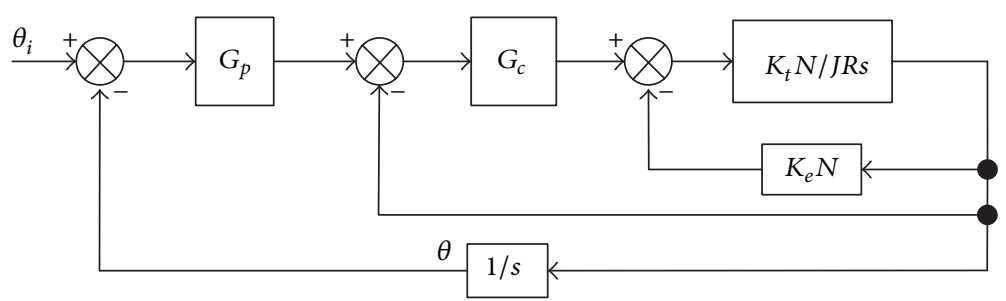

FIGURE 9: The block diagram of the single-axis GSP control system without phase compensation and disturbance.
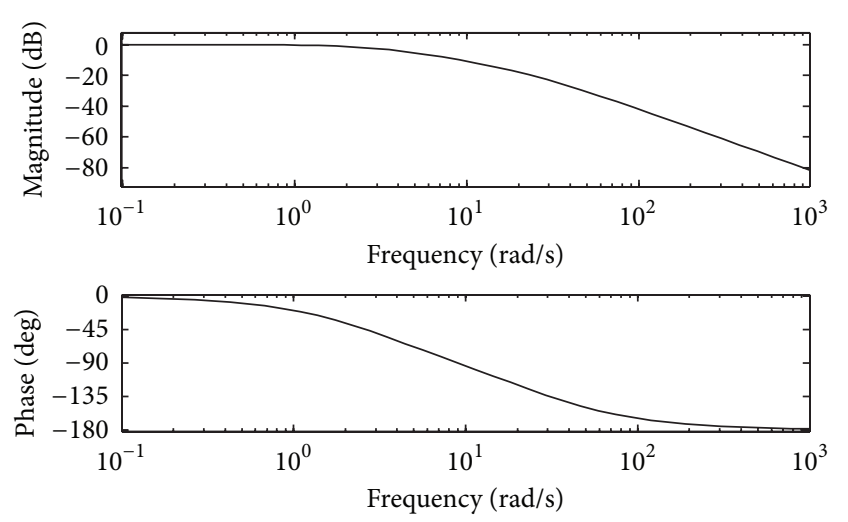

FIgURE 10: The transfer function $\left(\theta / \theta_{i}\right)$ frequency response.

The following adaptive nonlinear control law is proposed shown in Figure 12:

$$
\begin{aligned}
u=\frac{R}{K_{t} N}\left(J \ddot{\theta}_{i}\right. & +J G_{p} \dot{\theta}_{i}+\left(\frac{K_{e} K_{t} N^{2}}{R}-J G_{p}\right) \omega \\
& \left.+G_{c} e_{2}-\widehat{T}_{d}-\widehat{W}^{T} \phi(x)+\widehat{\mu} \operatorname{sgn}\left(e_{2}\right)\right),
\end{aligned}
$$

where $\widehat{\mu}$ is the estimated value of $\varepsilon_{M}$.

Substituting (10) and (8) into (6),

$$
\begin{aligned}
J \dot{e}_{2} & =J \ddot{\theta}_{i}+J G_{p} \dot{\theta}_{i}+\left(\frac{K_{e} K_{t} N^{2}}{R}-J G_{p}\right) \omega-\frac{K_{t} N}{R} u-T_{d} \\
& =-\left(G_{c} e_{2}-\widehat{T}_{d}-\widehat{W}^{T} \phi(x)+\widehat{\mu} \operatorname{sgn}\left(e_{2}\right)\right)-T_{d} \\
& =-\left(G_{c} e_{2}-\widehat{T}_{d}-\widehat{W}^{T} \phi(x)+\widehat{\mu} \operatorname{sgn}\left(e_{2}\right)\right)-T_{d} \\
& =-\left(G_{c} e_{2}-\widehat{W}^{T} \phi(x)+\widehat{\mu} \operatorname{sgn}\left(e_{2}\right)\right)-\left(T_{d}-\widehat{T}_{d}\right) \\
& =-\left(G_{c} e_{2}-\widehat{W}^{T} \phi(x)+\widehat{\mu} \operatorname{sgn}\left(e_{2}\right)\right)-W^{* T} \phi(x)+\varepsilon \\
& =-G_{c} e_{2}-\left(W^{* T} \phi(x)-\widehat{W}^{T} \phi(x)\right)+\varepsilon-\widehat{\mu} \operatorname{sgn}\left(e_{2}\right) \\
& =-G_{c} e_{2}-\widetilde{W}^{T} \phi(x)+\varepsilon-\widehat{\mu} \operatorname{sgn}\left(e_{2}\right),
\end{aligned}
$$

where

$$
\widetilde{W}=W^{*}-\widehat{W}
$$

is the weight-estimation errors.
The adaptation laws for the parameters $\widehat{W}$ and $\widehat{\mu}$ are

$$
\begin{gathered}
\dot{\widehat{W}}=-\delta e_{2} \phi(x) \\
\dot{\hat{\mu}}=\gamma e_{2} \operatorname{sgn}\left(e_{2}\right),
\end{gathered}
$$

where $\delta>0$ is a dimensionally compatible constant matrix, and $\gamma>0$.

Then, the stability of the proposed scheme can be analyzed by Lyapunov criterion as follows.

Theorem 1. Assuming that the angle commands $\theta_{i}, \dot{\theta}_{i}$, and $\ddot{\theta}_{i}$ are bounded, the control laws (10), together with the parameter update laws (13), guarantee the tracking errors $e_{1}$ and $e_{2}$ to be uniformly ultimately bounded.

Proof. Consider the following Lyapunov function:

$$
V=\frac{1}{2} J e_{2}^{2}+\frac{1}{2 \delta} \widetilde{W}^{T} \widetilde{W}+\frac{1}{2 \gamma} \widetilde{\mu}^{2},
$$

where $\tilde{\mu}=\varepsilon_{M}-\widehat{\mu}$.

By applying (11)-(14), the time derivative of $V$ is given by

$$
\begin{aligned}
\dot{V}= & e_{2} J \dot{e}_{2}+\frac{1}{\delta} \widetilde{W}^{T} \dot{\widetilde{W}}+\frac{1}{\gamma} \tilde{\mu} \dot{\tilde{\mu}} \\
= & -G_{c} e_{2}^{2}-\widetilde{W}^{T} \phi(x) e_{2}+\varepsilon e_{2}-\widehat{\mu} e_{2} \operatorname{sgn}\left(e_{2}\right) \\
& +\frac{1}{\delta} \widetilde{W}^{T} \dot{\widetilde{W}}+\frac{1}{\gamma} \tilde{\mu} \dot{\tilde{\mu}} \\
= & -G_{c} e_{2}^{2}+\widetilde{W}^{T}\left(\frac{1}{\delta} \dot{\widetilde{W}}-\phi(x) e_{2}\right)+\varepsilon e_{2} \\
& -\widehat{\mu} e_{2} \operatorname{sgn}\left(e_{2}\right)+\frac{1}{\gamma} \tilde{\mu} \dot{\tilde{\mu}} \\
\leq & -G_{c} e_{2}^{2}+\varepsilon_{M} e_{2} \operatorname{sgn}\left(e_{2}\right)-\widehat{\mu} e_{2} \operatorname{sgn}\left(e_{2}\right)-\widetilde{\mu} e_{2} \operatorname{sgn}\left(e_{2}\right) \\
\leq & -G_{c} e_{2}^{2} \\
\leq & 0 .
\end{aligned}
$$

From Lyapunov stability theory, the Lyapunov function $V$ is an energy function of errors $e_{2}, \widetilde{W}$, and $\widetilde{\mu}$, and $\dot{V} \leqslant 0$, which is derived from expression (15). Therefore, the errors can then asymptotically converge to zero as time goes to infinity. So, for GSP system in this paper, the designed control system is stable 


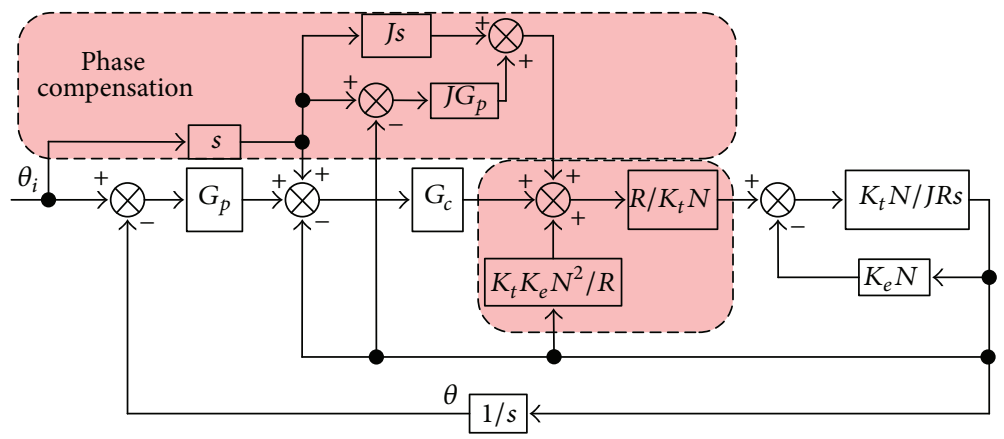

FIGURE 11: Phase compensation for the single-axis GSP control system.

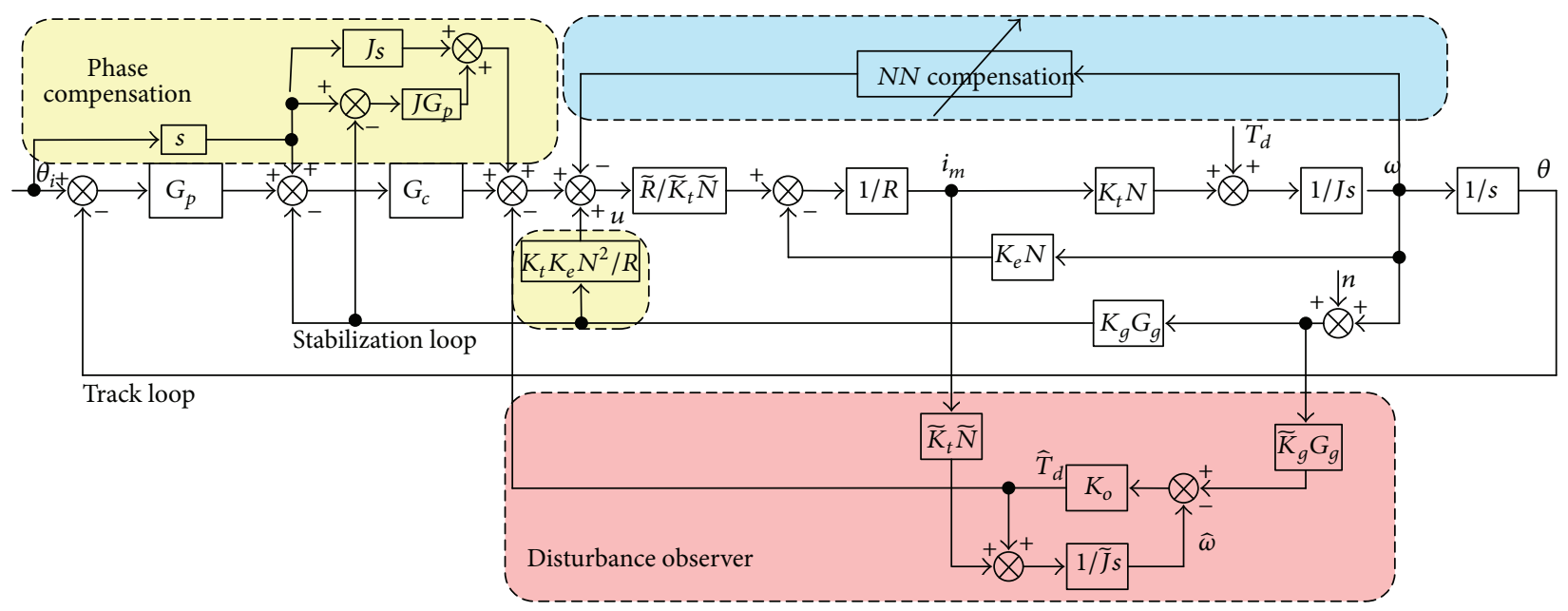

FIGURE 12: An adaptive nonlinear control for the single-axis GSP system.

and the variables $e_{2}$ will converge to a neighborhood of zero in a finite time. Because $e_{2}=\dot{e}_{1}+G_{p} e_{1}$ is a stable system, the tracking error will also converge to a neighborhood of zero by adjusting some control design parameters.

\section{Experiments}

4.1. Experimental Setup. To demonstrate the effectiveness of the proposed method, real-time experiments are carried out on the outer gimbal of GSP shown in Figure 13. The gimbal position is measured by accelerometer, dc current is measured by high bandwidth Hall effect current sensor, and the angle velocity of the gimbal is measured by fiber optic gyroscope and fed to a controller through analog-todigital converter. The controller, which is programmed using a DSP TMS320F28335 with $30 \mathrm{MHz}$ oscillator, is employed to generate necessary control input signals for high tracking and stabilized precision in the condition of unknown disturbance torque. The sampling period for our test is chosen as $0.01 \mathrm{~s}$. The parameters of the single-axis GSP control system are listed in Table 1.

4.2. Adaptive Nonlinear Controller. In order to avoid the well-known chattering phenomenon in actual experiment,

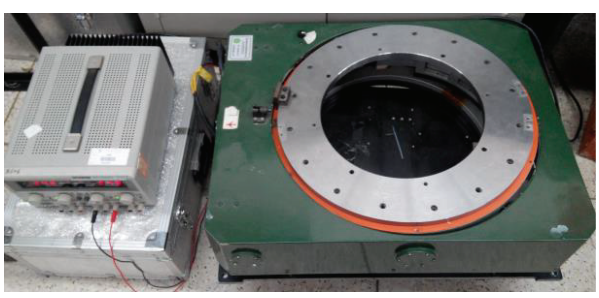

FIgURE 13: Experimental setup.

saturation function shown in (16) is used to replace the sign function in the control law (10):

$$
\text { sat }\left(e_{2}\right)= \begin{cases}1, & \left(e_{2}>\eta\right), \\ \frac{1}{\eta} e_{2}, & \left(\left|e_{2}\right| \leq \eta\right), \\ -1, & \left(e_{2}<\eta\right),\end{cases}
$$

where $\eta$ is a small positive content.

The radial basis function (RBF) NN is selected as the NN learning model as follows:

$$
\varphi_{i}(x)=\exp \left(-\frac{\left\|x-c_{i}\right\|^{2}}{\sigma_{i}^{2}}\right), \quad i=1,2, \ldots, N .
$$




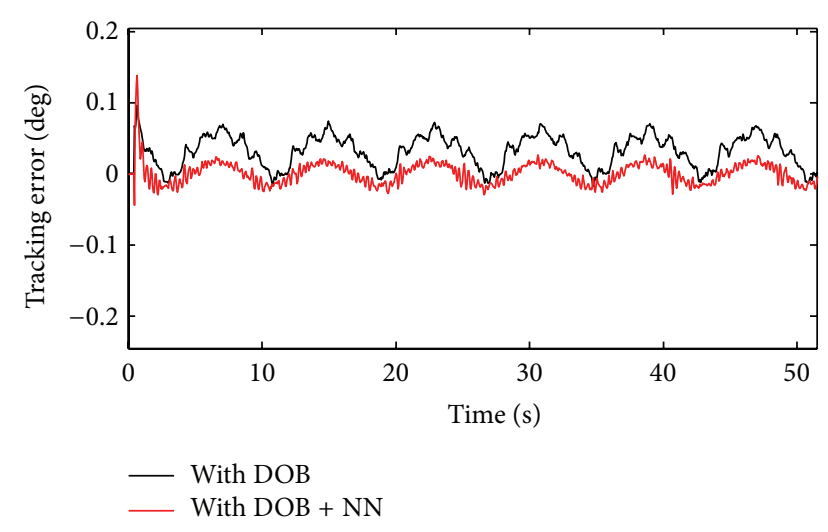

FIGURE 14: The tracking error of the single-axis GSP system for 0.125 $\mathrm{Hz}$ sinusoidal references of amplitude 1 degree with two methods.

The RBF NN contains 5 nodes with centers $c_{i}(i=$ $1,2, \ldots N)$ evenly spaced in $[-0.015,0.015]$ and widths $\sigma_{i}=$ $0.5(i=1,2, \ldots N)$. The $\mathrm{NN}$ weights $\widehat{W}$ are simply initialized at zero, and the initial value of $\hat{\mu}$ is selected as 0.01 . The adaptation laws for adjusting the controller are given by (13) with the adaptation rates $\delta=15$ and $\gamma=0.05$. During the experimental test, the parameters $G_{p}$ and $G_{c}$ in the control law (10) are chosen as 3 and 5.5, respectively. The gain of reduced-order disturbance observer $K_{o}=20$; the parameter of saturation function $\eta=0.05$.

Remark 2. The parameters have effects on the system performance. Theoretically, the larger the $K_{o}$, the smaller the tracking error; however, $K_{o}$ is limited by the rigidity of the mechanism, since large $K_{o}$ may lead to the system vibration. If $\delta$ is too small, the convergence of the neural weights is slow, which will degrade the system precision. However, if it is large, the convergence may be too fast and leads to undesirable transient response because of large overshoot. So, these parameter values should be chosen by trial-and-error until good performance is obtained.

(1) Tracking Experiment. The efficacy of the proposed controller in reducing the tracking error is first analyzed. The control objective is to follow a sine wave signal $\theta_{i}=$ $\pi \sin (2 \pi t / 8) / 180 \mathrm{rad}$. From Figure 14 it is found that the tracking error using DOB $+\mathrm{NN}$ decreases by $60 \%$ compared with the controller only with DOB. Figure 15 shows the estimated disturbance torque with the two methods.

(2) Stabilizing Experiment. Next, the efficacy of the proposed controller in improving the stabilizing accuracy is analyzed. The desired position $\theta_{i}$ is zero. A constant disturbance is added at $t=84 \mathrm{~s}$ and is cancelled after $100 \mathrm{~s}$. Figure 16 shows the tracking error of the single-axis GSP system with two methods. The method with DOB has obvious error when adding a constant disturbance, while the method with DOB + NN has more disturbance suppression ability since the tracking error stays almost unchanged. Figure 17 shows the estimated disturbance torque with two methods.

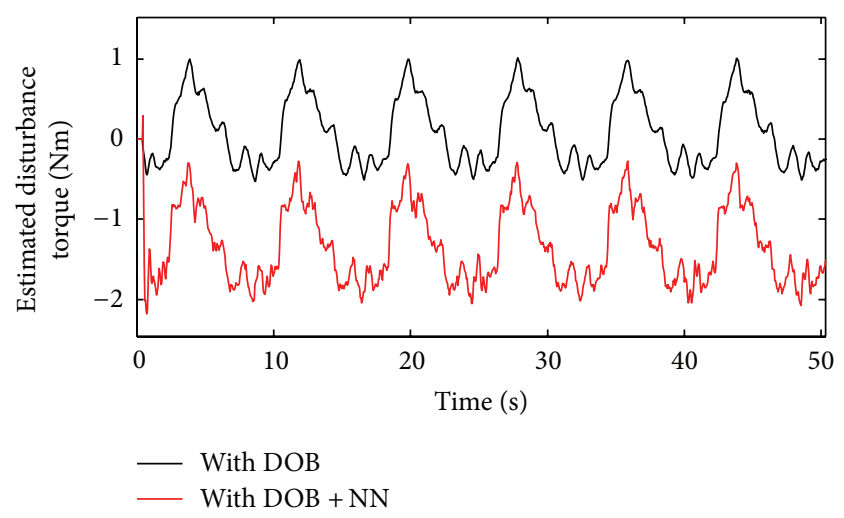

FIGURE 15: The estimated disturbance torque with two methods.

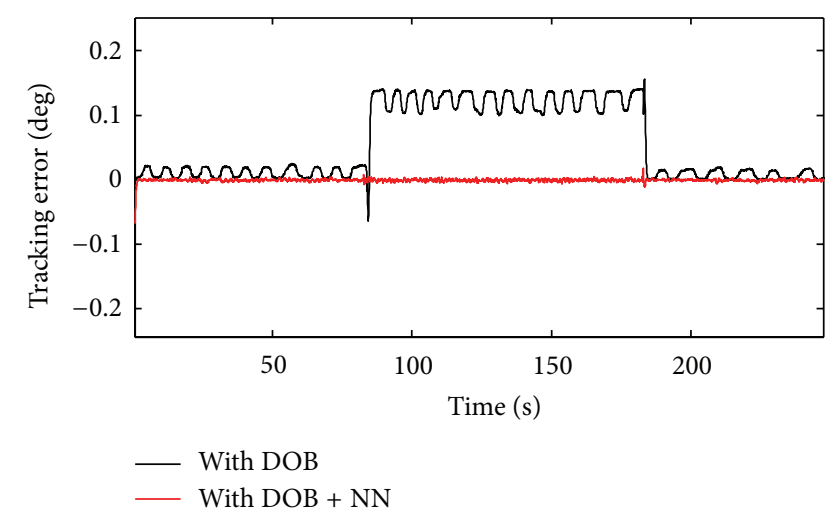

FIgURE 16: The tracking error of the single-axis GSP system with two methods.

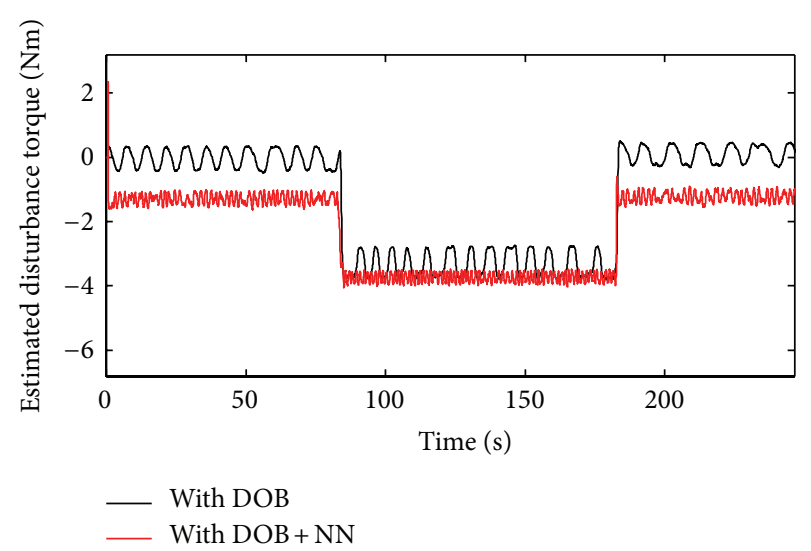

FIGURE 17: The estimated disturbance torque with two methods.

It is thus readily concluded that the proposed controller can reduce the effect of external disturbance and significantly improve the tracking and stabilizing accuracy, for more accurate estimate of the disturbance of the GSP system is compensated with the proposed controller. 


\section{Conclusion}

The reduced-order disturbance observer and neural networks compensator are used to design an adaptive nonlinear controller for disturbance compensation. The disturbance is estimated by the reduced-order disturbance observer, and the neural networks compensator based on radial basis function approximates the error of estimated disturbance. It is found that the tracking error using the proposed method has been reduced by $60 \%$ compared with the conventional DOB, and the method with $\mathrm{DOB}+\mathrm{NN}$ also has more disturbance suppression ability compared with DOB.

\section{Conflict of Interests}

The authors declare that there is no conflict of interests regarding the publication of this paper.

\section{Acknowledgments}

This work was supported by Chinese National Innovation Community Foundation (nos. 61121003, 61273033, and 61473019) and the National Natural Science Foundation of China (no. 51205019).

\section{References}

[1] J. M. Hilkert, "Inertially stabilized platform technology: concepts and principles," IEEE Control Systems Magazine, vol. 28, no. 1, pp. 26-46, 2008.

[2] M. K. Masten, "Inertially stabilized platforms for optical imaging systems," IEEE Control Systems, vol. 28, no. 1, pp. 47-64, 2008.

[3] H. G. Wang and T. C. Williams, "Strategic inertial navigation systems-high-accuracy inertially stabilized platforms for hostile environments," IEEE Control Systems, vol. 28, no. 1, pp. 6585, 2008.

[4] J. L. Miller, S. Way, B. Ellison, and C. Archer, "Design challenges regarding high-definition electro-optic/infrared stabilized imaging systems," Optical Engineering, vol. 52, no. 6, Article ID 061310, 2013.

[5] R. Miller, G. Mooty, and J. M. Hilkert, "Gimbal system configurations and line-of-sight control techniques for small UAV applications," in Airborne Intelligence, Surveillance, Reconnaissance (ISR) Systems and Applications X, vol. 8713 of Proceedings of SPIE, pp. 1-15, 2013.

[6] J. Hilkert and D. L. Amil, "Structural effects and techniques in precision pointing and tracking systems-a tutorial overview," in Automatic Target Recognition XX; Acquisition, Tracking, Pointing, and Laser Systems Technologies XXIV; and Optical Pattern Recognition XXI, vol. 7696 of Proceedings of SPIE, 76961C, p. 1-12, 2010.

[7] J. M. Hilkert and B. Pautler, "A reduced-order disturbance observer applied to inertially stabilized Line-of-Sight control," in 25th Acquisition, Tracking, Pointing, and Laser Systems Technologies, vol. 8052 of Proceedings of SPIE, p. $80520 \mathrm{H}$, Orlando, Fla, USA, April 2011.

[8] C. Wu and Z. Lin, "Disturbance observer based control system design for inertially stabilized platform," in Electro-Optical Remote Sensing, Photonic Technologies, and Applications VI,
85420T, vol. 8542 of Proceedings of SPIE, Edinburgh, UK, September 2012.

[9] A. Li, C. Hong, S. Zhang, and C. Yuan, "High-precision stabilization control for a floated inertial platform," in Proceedings of the 25th Chinese Control and Decision Conference (CCDC '13), pp. 1193-1199, May 2013.

[10] Y. Hu, Y. Cao, and S. Zhang, "Design of sliding mode control with disturbance observers for inertial platform," in Proceedings of the 25th Chinese Control and Decision Conference (CCDC '13), pp. 4652-4656, May 2013.

[11] S. Mondal, S. Sadhu, and A. Banerjee, "Platform motion disturbances attenuation in a missile seeker subsystem using Internal Model Control," in Proceedings of the International Conference on Control, Automation, Robotics and Embedded Systems (CARE '13), pp. 1-4, Jabalpur, India, December 2013.

[12] Z. Yang, J. Wu, and J. Mei, "Motor-mechanism dynamic model based neural network optimized computed torque control of a high speed parallel manipulator," Mechatronics, vol. 17, no. 7, pp. 381-390, 2007.

[13] X. Sun, L. Chen, Z. Yang, and H. Zhu, "Speed-sensorless vector control of a bearingless induction motor with artificial neural network inverse speed observer," IEEE/ASME Transactions on Mechatronics, vol. 18, no. 4, pp. 1357-1366, 2013.

[14] J.-F. Couchot, K. Deschinkel, and M. Salomon, "Active MEMSbased flow control using artificial neural network," Mechatronics, vol. 23, no. 7, pp. 898-905, 2013.

[15] X. Ren, C. Y. Lai, V. Venkataramanan, F. L. Lewis, S. S. Ge, and T. Liew, "Feedforward control based on neural networks for disturbance rejection in hard disk drives," IET Control Theory \& Applications, vol. 3, no. 4, pp. 411-418, 2009.

[16] K. K. Ahn and H. P. H. Anh, "Design and implementation of an adaptive recurrent neural networks (ARNN) controller of the pneumatic artificial muscle (PAM) manipulator," Mechatronics, vol. 19, no. 6, pp. 816-828, 2009. 


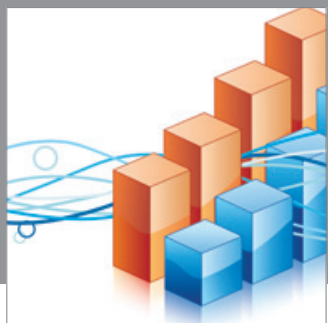

Advances in

Operations Research

mansans

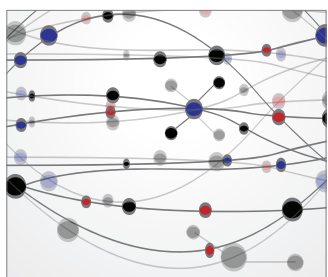

The Scientific World Journal
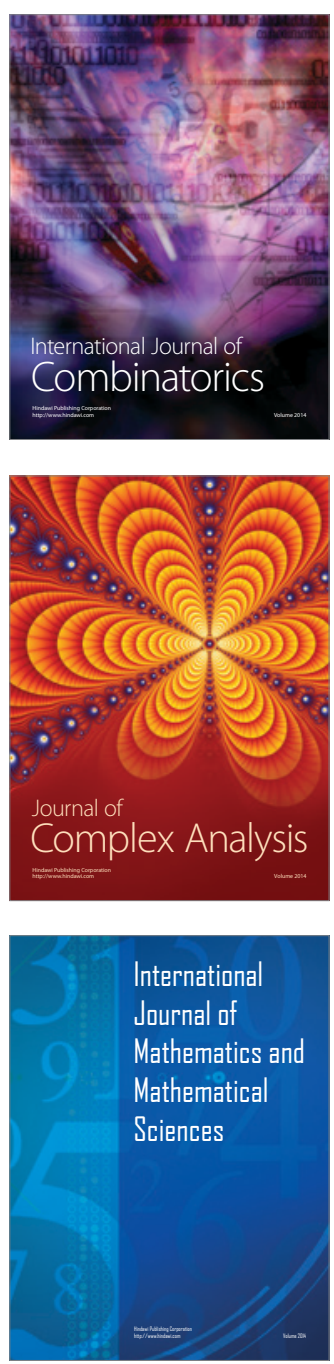
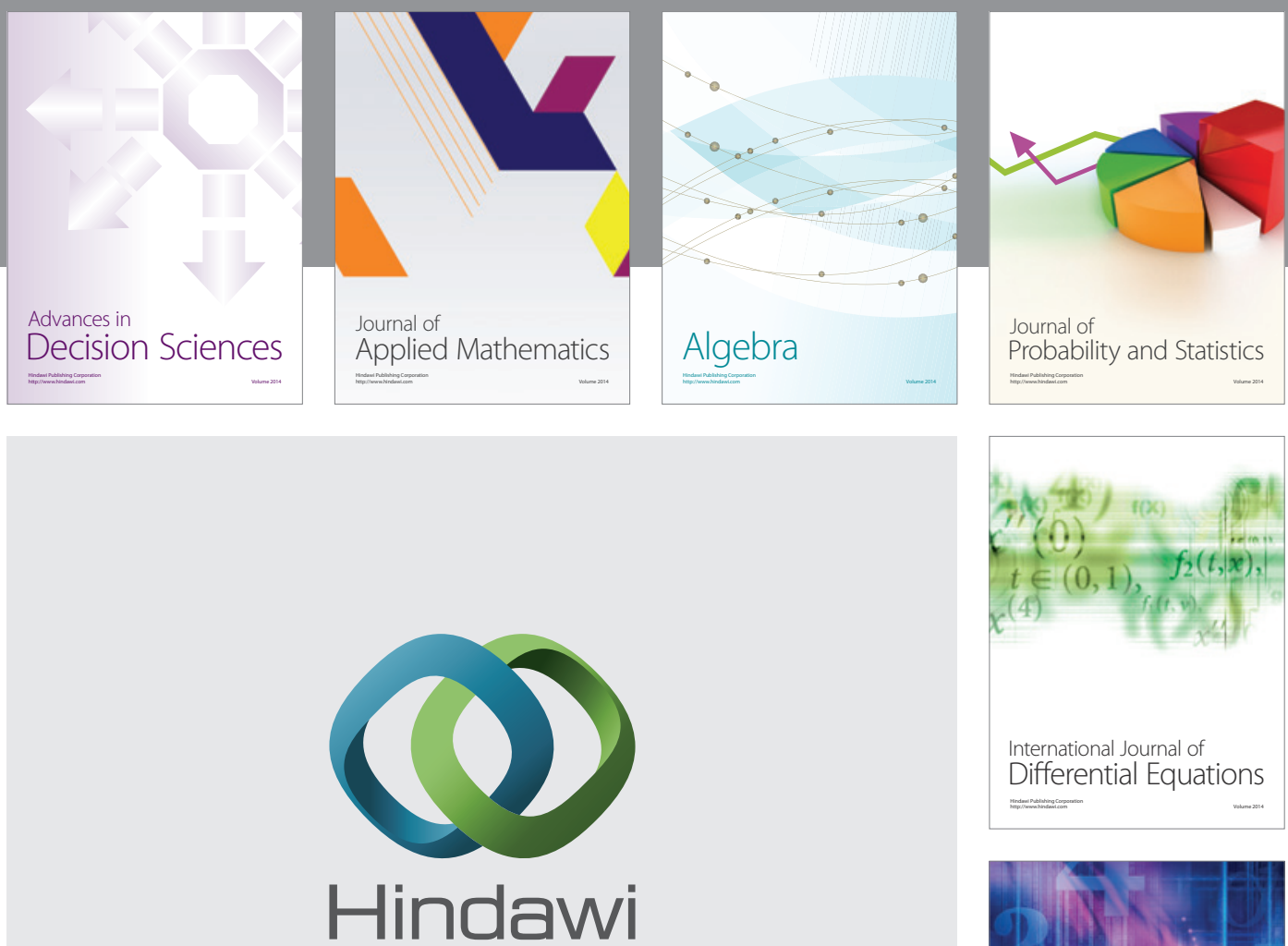

Submit your manuscripts at http://www.hindawi.com
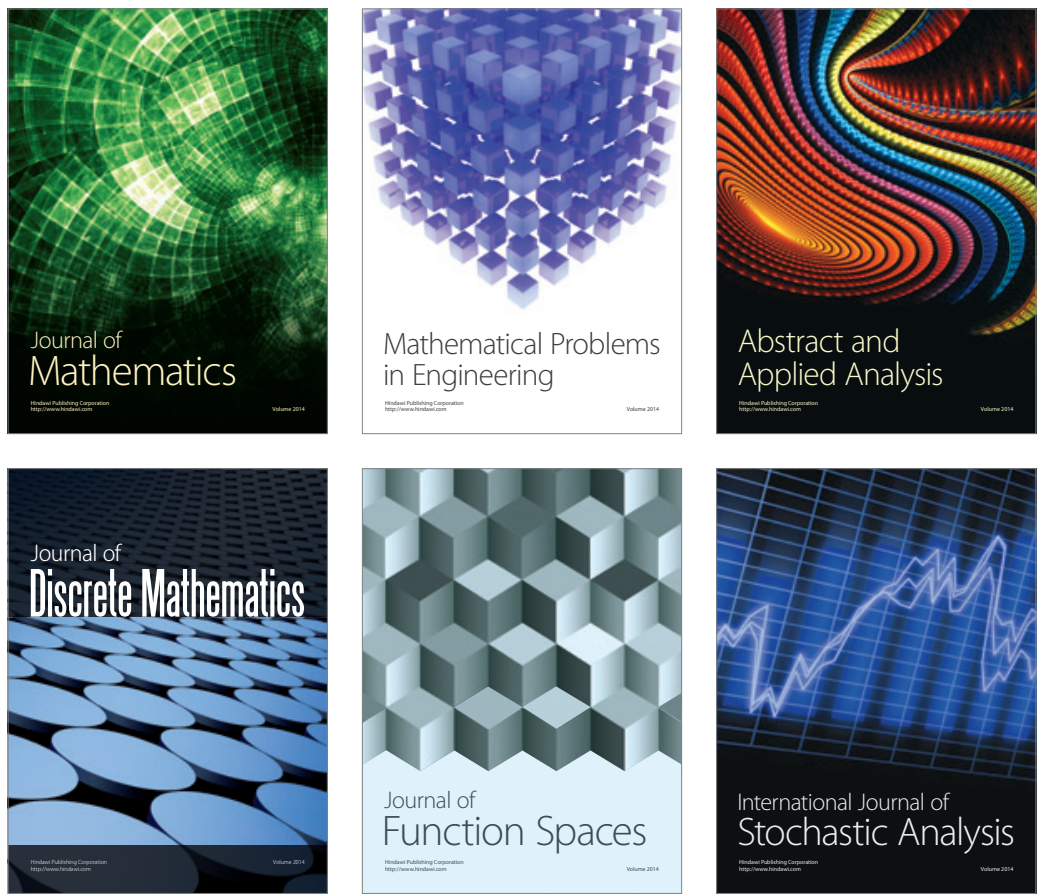

Journal of

Function Spaces

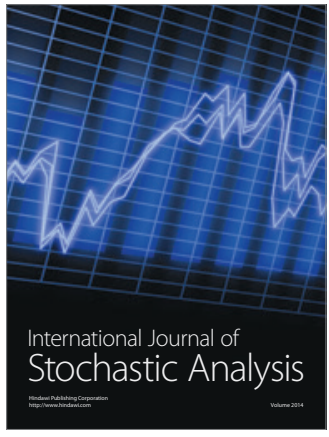

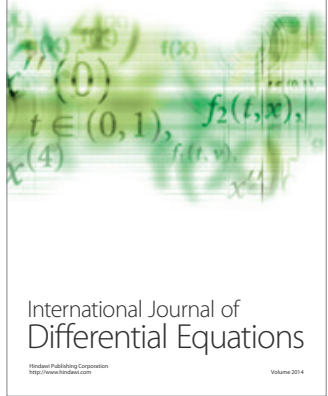
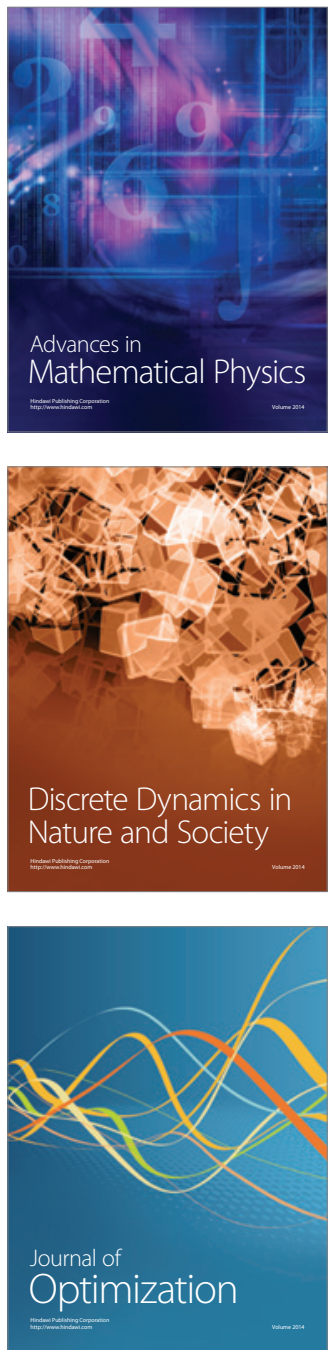